\title{
On Non-Vanishing of Convolution of Dirichlet Series
}

\author{
Amir Akbary*, Shahab Shahabi ${ }^{\dagger}$
}

\begin{abstract}
We study the non-vanishing on the line $\operatorname{Re}(s)=1$ of the convolution series associated to two Dirichlet series in a certain class of Dirichlet series. The non-vanishing of various $L$-functions on the line $\operatorname{Re}(s)=1$ will be simple corollaries of our general theorems.
\end{abstract}

Let $f(z)=\sum_{n=1}^{\infty} \hat{a}_{f}(n) e^{2 \pi i n z}$ and $g(z)=\sum_{n=1}^{\infty} \hat{a}_{g}(n) e^{2 \pi i n z}$ be cusp forms of weight $k$ and level $N$ with trivial character. Let $L_{f}(s)=\sum_{n=1}^{\infty} a_{f}(n) n^{-s}$ and $L_{g}(s)=\sum_{n=1}^{\infty} a_{g}(n) n^{-s}$ be the $L$-functions associated to $f$ and $g$, respectively, where $a_{f}(n)=\hat{a}_{f}(n) / n^{\frac{k-1}{2}}$ and $a_{g}(n)=$ $\hat{a}_{g}(n) / n^{\frac{k-1}{2}}$. Let

$$
L(f \otimes g, s)=\zeta_{N}(2 s) \sum_{n=1}^{\infty} \frac{a_{f}(n) \overline{a_{g}(n)}}{n^{s}}
$$

be the Rankin-Selberg convolution of $L_{f}(s)$ and $L_{g}(s)$. In [11] Rankin established the analytic continuation of $L(f \otimes g, s)$ (see Theorem 1.5). Rankin's Theorem has numerous number theoretic applications. In [10], Rankin used this theorem to prove the non-vanishing of the modular $L$ function associated to the discriminant function

$$
\Delta(z)=e^{2 \pi i z} \prod_{n=1}^{\infty}\left(1-e^{2 \pi i n z}\right)^{24}
$$

on the line $\operatorname{Re}(s)=1$. In fact, Rankin's argument establishes the non-vanishing of $L$-functions associated to eigenforms for the points on the line $R e(s)=1$, except the point $s=1$. In [9], Ogg proved that the same result is true for $s=1$. Moreover, he showed the following.

Theorem $0.1(\mathrm{Ogg})$ If $f$ and $g$ are eigenforms with respect to the family of the Hecke operators for $\Gamma_{0}(N)$ and $\langle f, g\rangle=0$, then $L(f \otimes g, 1) \neq 0$. Here $\langle f, g\rangle$ denote the Petersson inner product of $f$ and $g$.

${ }^{0} 1991$ Mathematics Subject Classification. Primary 11F67.

${ }^{*}$ Research partially supported by NSERC.

${ }^{\dagger}$ Research partially supported by NSERC. 
In this paper we prove similar non-vanishing results (Theorem 2.3, Theorem 3.5 and Theorem 4.2) for the convolution of two Dirichlet series belonging to a certain family of Dirichlet series $\mathcal{S}^{*}$ (see Definitions 1.1 and 1.2). Our theorems are quite general and clearly demonstrate the close connection between the analytic continuation of a Dirichlet series and its various convolutions to the left of its half plane of convergence and its non-vanishing on the line $\operatorname{Re}(s)=1$. More precisely, for two Dirichlet series $F$ and $G \in \mathcal{S}^{*}$ with Euler products

$$
F(s)=\prod_{p} \exp \left(\sum_{k=1}^{\infty} \frac{b_{F}\left(p^{k}\right)}{p^{k s}}\right) \text { and } G(s)=\prod_{p} \exp \left(\sum_{k=1}^{\infty} \frac{b_{G}\left(p^{k}\right)}{p^{k s}}\right)
$$

valid on $\operatorname{Re}(s)>1$, we define the Euler product convolution of $F$ and $G$ as

$$
(F \otimes G)(s)=\prod_{p} \exp \left(\sum_{k=1}^{\infty} \frac{k b_{F}\left(p^{k}\right) \overline{b_{G}\left(p^{k}\right)}}{p^{k s}}\right) .
$$

We say $F \in \mathcal{S}^{*}$ is $\otimes$-simple in $\operatorname{Re}(s) \geq \sigma_{0}$, if $F \otimes F$ has an analytic continuation to $\operatorname{Re}(s) \geq \sigma_{0}$, except for a possible simple pole at $s=1$. One of our main results is the following.

Theorem 2.3 Let $F, G \in \mathcal{S}^{*}$ be $\otimes$-simple in $\operatorname{Re}(s) \geq 1$ and $t \neq 0$. Then

(i) $(F \otimes F)(1+i t) \neq 0$.

(ii) If $F \otimes G$ has an analytic continuation to the line $\operatorname{Re}(s)=1$ and $(F \otimes G)(s)=0$ if and only if $(F \otimes G)(\bar{s})=0$ for any $s$ on the line $\operatorname{Re}(s)=1$, then $(F \otimes G)(1+i t) \neq 0$.

Note that this result does not say anything about the value of $(F \otimes G)(s)$ at $s=1$. to deal with this case, in Section 3 we prove a non-vanishing theorem, valid on the line $\operatorname{Re}(s)=1$, for Euler product convolution of two Dirichlet series in $\mathcal{S}^{*}$ with completely multiplicative coefficients (Theorem 3.5). Finally in Section 4 for Dirichlet series with general coefficients we prove the following.

Theorem 4.2 Let $\sigma_{0}<1$, and assume the following:

(i) $F$ and $G$ (as elements of $\mathcal{S}^{*}$ ) are $\otimes$-simple in $\operatorname{Re}(s)>\sigma_{0}$;

(ii) $F \otimes G$ has an analytic continuation to the half-plane $\operatorname{Re}(s)>\sigma_{0}$;

(iii) At least one of $F \otimes F, G \otimes G$, or $F \otimes G$ has zeros in the strip $\sigma_{0}<\operatorname{Re}(s)<1$. Then $(F \otimes G)(1+i t) \neq 0$ for all real $t$.

Our general theorems have several applications. The non-vanishing of various classical $L$ functions will be simple corollaries of our general theorems (see Corollaries 2.4, 2.6 and 4.4). Moreover, as a consequence of our theorems, we will be able to extend Ogg's theorem to the line $\operatorname{Re}(s)=1$ (Corollary 2.6, (iv)). Another application will result in an extension of Ogg's nonvanishing result to the line $R e(s)=1$ and for eigenforms with characters (Corollary 4.4, (iv)). Corollary 4.4, (ii) gives a generalization of the non-vanishing result of Rankin to eigenforms with characters. Finally non-vanishing of twisted symmetric square $L$-functions on the line $\operatorname{Re}(s)=1$ 
(Corollary 4.4, $(v)$ ) is a simple consequence of our theorems. Our general theorems could also be applied to the $L$-functions associated to number fields, however, in applications of this paper we restrict ourselves to Dirichlet and modular $L$-functions. For results of these types in the context of automorphic forms and representations see [4], [12] and [13].

Our approach in this paper is motivated by [8] and section 8.4 of [6].

Notation In this paper we use the following notations:

$\zeta(s)=\sum_{n=1}^{\infty} 1 / n^{s}:$ the Riemann zeta-function,

$\zeta_{q}(s)=\prod_{p \mid q}(1-1 / p) \zeta(s)$ : the Riemann zeta-function with the Euler $p$-factors corresponding to $p \mid N$ removed,

$L_{\chi}(s)=\sum_{n=1}^{\infty} \chi(n) / n^{s}$ : the Dirichlet $L$-function associated to a Dirichlet character $\chi$,

$S_{k}(N)$ : the space of cusp forms of weight $k$ and level $N$ with the trivial character,

$S_{k}(N, \psi)$ : the space of cusp forms of weight $k$ and level $N$ with character $\psi$ where $\psi(-1)=(-1)^{k}$,

$\langle f, g\rangle=\int_{D_{0}(N)} f(z) \overline{g(z)} y^{k-2} d x d y:$ the Petersson inner product of $f, g \in S_{k}(N, \psi)$. Here, $D_{0}(N)$ is a fundamental domain for the congruence subgroup $\Gamma_{0}(N)$,

$L_{f}(s)=\sum_{n=1}^{\infty} a_{f}(n) / n^{s}$ : the $L$-function associated to a cusp form $f \in S_{k}(N, \psi)$,

$L_{f, \chi}(s)=\sum_{n=1}^{\infty} a_{f}(n) \chi(n) / n^{s}$ : the twisted $L$-function associated to a cusp form $f \in$ $S_{k}(N, \psi)$ and a Dirichlet character $\chi$,

$L(f \otimes g, s)=L_{\psi_{1} \bar{\psi}_{2}}(2 s) \sum_{n=1}^{\infty} a_{f}(n) \overline{a_{g}(n)} / n^{s}$ : the Rankin-Selberg convolution of $L_{f}(s)$ and $L_{g}(s)$, where $f \in S_{k}\left(N, \psi_{1}\right)$ and $g \in S_{k}\left(N, \psi_{2}\right)$,

$L\left(\operatorname{sym}^{2} f, s\right)=L(f \otimes f, s) / \zeta_{N}(s)$ : the symmetric square $L$-function associated to a normalized eigenform $f$ in $S_{k}(N)$,

$L_{\chi}(f \otimes g, s)=L_{\psi_{1} \bar{\psi}_{2} \chi^{2}}(2 s) \sum_{n=1}^{\infty} a_{f}(n) \overline{a_{g}(n)} \chi(n) / n^{s}$ : the twisted Rankin-Selberg convolution of $L_{f}(s)$ and $L_{g}(s)$, where $f \in S_{k}\left(N, \psi_{1}\right), g \in S_{k}\left(N, \psi_{2}\right)$ and $\chi$ is a Dirichlet character,

$L_{\chi}\left(\operatorname{sym}^{2} f, s\right)=L_{\chi}(f \otimes \bar{f}, s) / L_{\psi \chi}(s)$ : the twisted symmetric square $L$-function associated to a normalized eigenform $f$ with character $\psi$ and a Dirichlet character $\chi$.

Note that in the above definitions, we assume that $\operatorname{Re}(s)>1$ and for a normalized eigenform $f$ we have $a_{f}(1)=1$.

\section{A Class of Dirichlet Series}

We consider the following class of Dirichlet Series.

Definition 1.1 The class $\mathcal{S}^{* 1}$ is the family of Dirichlet series $F(s)=\sum_{n=1}^{\infty} a_{F}(n) n^{-s}(\operatorname{Re}(s)>$ 1) satisfying the following properties:

\footnotetext{
${ }^{1}$ We use this notation to emphasis the relation of this class to the Selberg class $\mathcal{S}$. Note that $\mathcal{S} \subset \mathcal{S}^{*}$. For the definition of the Selberg class $\mathcal{S}$ see [6], Chapter 8.
} 
(a) (Euler Product): For $\operatorname{Re}(s)>1$, we have

$$
F(s)=\prod_{p} \exp \left(\sum_{k=1}^{\infty} \frac{b_{F}\left(p^{k}\right)}{p^{k s}}\right) ;
$$

(b) (Ramanujan's Hypothesis): For any fixed $\epsilon>0$,

$$
a_{F}(n)=O\left(n^{\epsilon}\right)
$$

where the implied constant may depend upon $\epsilon$.

(c) (Analytic Continuation): $F(s)$ has an analytic continuation to the line $\operatorname{Re}(s)=1$, except for a possible pole at point $s=1$.

For $F \in \mathcal{S}^{*}$, we define

$$
\bar{F}(s)=\overline{F(\bar{s})}=\sum_{n=1}^{\infty} \frac{\overline{a_{F}(n)}}{n^{s}}=\prod_{p} \exp \left(\sum_{k=1}^{\infty} \frac{\overline{b_{F}\left(p^{k}\right)}}{p^{k s}}\right) .
$$

We continue by defining a convolution operation on $\mathcal{S}^{*}$.

Definition 1.2 For $F, G \in \mathcal{S}^{*}$, the Euler product convolution of $F$ and $G$ is defined as

$$
(F \otimes G)(s)=\prod_{p} \exp \left(\sum_{k=1}^{\infty} \frac{k b_{F}\left(p^{k}\right) \overline{b_{G}\left(p^{k}\right)}}{p^{k s}}\right) .
$$

The following lemma shows that this operation is well-defined on the half plane $\operatorname{Re}(s)>1$.

Lemma 1.3 For $F, G$ in $\mathcal{S}^{*},(F \otimes G)(s)$ is convergent for $\operatorname{Re}(s)>1$.

Proof Let $\epsilon>0$. One can show that $\left|a_{F}(n)\right| \leq c(\epsilon) n^{\epsilon}$ implies

$$
\left|b_{F}\left(p^{k}\right)\right| \leq \frac{c(\epsilon)\left(2^{k}-1\right) p^{k \epsilon}}{k}
$$

(see [6] Exercise 8.2.9). Now suppose that $\sigma=R e(s) \geq 1+3 \epsilon$. By applying (1) and the expansion

$$
-\log (1-z)=z+\frac{z^{2}}{2}+\frac{z^{3}}{3}+\cdots
$$

valid for $|z|<1$, we have

$$
\exp \left(\sum_{k=1}^{\infty} \frac{k b_{F}\left(p^{k}\right) \overline{b_{G}\left(p^{k}\right)}}{p^{k s}}\right) \ll \exp \left(\sum_{k=1}^{\infty} \frac{\left(2^{k}-1\right)^{2} p^{2 k \epsilon} / k}{p^{k \sigma}}\right) \ll\left(1-\frac{4}{p^{\sigma-2 \epsilon}}\right)^{-1} .
$$

Since $\sigma-2 \epsilon \geq 1+\epsilon>1$, the product $\prod_{p}\left(1-\frac{4}{p^{\sigma-2 \epsilon}}\right)$ is convergent. 
The next lemma will enable us to express several classical $L$-functions of number theory as Euler product convolution of two simpler $L$-functions. This lemma plays an important role in the applications of our general theorems.

Lemma 1.4 (i) $\zeta(s)$ is in $\mathcal{S}^{*}$, and for any $F$ in $\mathcal{S}^{*}$, we have

$$
(F \otimes \zeta)(s)=F(s)
$$

(ii) For $F$ in $\mathcal{S}^{*}$, we have

$$
(\zeta \otimes F)(s)=\bar{F}(s) .
$$

(iii) If $\chi$ is a Dirichlet character $(\bmod q)$, then $L_{\chi}(s)$ is in $\mathcal{S}^{*}$, and

$$
\left(L_{\chi} \otimes L_{\chi}\right)(s)=\zeta_{q}(s)
$$

(iv) Let $f$ be a normalized eigenform in $S_{k}(N, \psi)$. Then $L_{f}(s)$ is in $\mathcal{S}^{*}$, and

$$
\left(L_{f} \otimes L_{\chi}\right)(s)=L_{f, \bar{\chi}}(s) .
$$

(v) For any two normalized eigenforms $f \in S_{k}\left(N, \psi_{1}\right)$ and $g \in S_{k}\left(N, \psi_{2}\right)$ and Dirichlet characters $\chi_{1}$ and $\chi_{2}, L_{f, \chi_{1}}(s)$ and $L_{g, \chi_{2}}(s)$ are in $\mathcal{S}^{*}$, and

$$
\left(L_{f, \chi_{1}} \otimes L_{g, \chi_{2}}\right)(s)=L_{\chi_{1} \bar{\chi}_{2}}(f \otimes g, s) .
$$

Proof We only prove the identity in part (v). Using (2) we have

$$
\begin{gathered}
L_{f, \chi_{1}}(s)=\prod_{p}\left(1-a_{f}(p) \chi_{1}(p) p^{-s}+\psi_{1}(p) \chi_{1}(p)^{2} p^{-2 s}\right)^{-1} \\
=\prod_{p}\left(1-\alpha_{f}(p) \chi_{1}(p) p^{-s}\right)^{-1}\left(1-\beta_{f}(p) \chi_{1}(p) p^{-s}\right)^{-1}=\prod_{p} \exp \left(\sum_{l=1}^{\infty} \frac{\left(\alpha_{f}(p)^{l}+\beta_{f}(p)^{l}\right) \chi_{1}(p)^{l} / l}{p^{l s}}\right)
\end{gathered}
$$

where $\alpha_{f}(p)+\beta_{f}(p)=a_{f}(p), \alpha_{f}(p) \beta_{f}(p)=\psi_{1}(p)$. We have also a similar product representation for $L_{g, \chi_{2}}(s)$. So

$$
\begin{aligned}
\left(L_{f, \chi_{1}}\right. & \left.\otimes L_{g, \chi_{2}}\right)(s)=\prod_{p} \exp \left(\sum_{l=1}^{\infty} \frac{\left(\alpha_{f}(p)^{l}+\beta_{f}(p)^{l}\right)\left(\overline{\alpha_{g}(p)}+\overline{\beta_{g}(p)}\right) \chi_{1}(p)^{l} \overline{\chi_{2}(p)} l l}{p^{l s}}\right) \\
= & \prod_{p}\left(1-\alpha_{f}(p) \overline{\alpha_{g}(p)} \chi_{1}(p) \overline{\chi_{2}(p)} p^{-s}\right)^{-1}\left(1-\alpha_{f}(p) \overline{\beta_{g}(p)} \chi_{1}(p) \overline{\chi_{2}(p)} p^{-s}\right)^{-1} \\
& \times\left(1-\beta_{f}(p) \overline{\alpha_{g}(p)} \chi_{1}(p) \overline{\chi_{2}(p)} p^{-s}\right)^{-1}\left(1-\beta_{f}(p) \overline{\beta_{g}(p)} \chi_{1}(p) \overline{\chi_{2}(p)} p^{-s}\right)^{-1} .
\end{aligned}
$$


Applying the identity $a_{f}\left(p^{l}\right)=a_{f}(p) a_{f}\left(p^{l-1}\right)-\psi_{1}(p) a_{f}\left(p^{l-2}\right)$ (and a similar one for the coefficients of $g$ ) repeatedly yields

$$
\begin{gathered}
a_{f}\left(p^{l}\right) \overline{a_{g}\left(p^{l}\right)}-a_{f}(p) a_{f}\left(p^{l-1}\right) \overline{a_{g}(p)} \overline{a_{g}\left(p^{l-1}\right)}+\left(\overline{\psi_{2}(p)} a_{f}(p)^{2}+\psi_{1}(p){\overline{a_{g}(p)}}^{2}-2 \psi_{1}(p) \overline{\psi_{2}(p)}\right) a_{f}\left(p^{l-2}\right) \overline{a_{g}\left(p^{l-2}\right)} \\
-\psi_{1}(p) \overline{\psi_{2}(p)} a_{f}(p) a_{f}\left(p^{l-3}\right) \overline{a_{g}(p)} \overline{a_{g}\left(p^{l-3}\right)}+\psi_{1}(p)^{2}{\overline{\psi_{2}(p)}}^{2} a_{f}\left(p^{l-4}\right) \overline{a_{g}\left(p^{l-4}\right)}=0 .
\end{gathered}
$$

Using this we arrive at

$$
\begin{aligned}
& \left(1-\psi_{1}(p) \overline{\psi_{2}(p)} \chi_{1}(p)^{2} \overline{\chi_{2}(p)} p^{-2 s}\right)^{-1} \sum_{l=0}^{\infty} \frac{a_{f}\left(p^{l}\right) \overline{a_{g}\left(p^{l}\right)} \chi_{1}\left(p^{l}\right) \overline{\chi_{2}\left(p^{l}\right)}}{p^{l s}} \\
= & \prod_{p}\left(1-\alpha_{f}(p) \overline{\alpha_{g}(p)} \chi_{1}(p) \overline{\chi_{2}(p)} p^{-s}\right)^{-1}\left(1-\alpha_{f}(p) \overline{\beta_{g}(p)} \chi_{1}(p) \overline{\chi_{2}(p)} p^{-s}\right)^{-1} \\
\times & \left(1-\beta_{f}(p) \overline{\alpha_{g}(p)} \chi_{1}(p) \overline{\chi_{2}(p)} p^{-s}\right)^{-1}\left(1-\beta_{f}(p) \overline{\beta_{g}(p)} \chi_{1}(p) \overline{\chi_{2}(p)} p^{-s}\right)^{-1} .
\end{aligned}
$$

Therefore

$$
\begin{gathered}
L_{\chi_{1} \bar{\chi}_{2}}(f \otimes g, s)=L_{\psi_{1} \bar{\psi}_{2} \chi_{1}{ }^{2} \bar{\chi}_{2}^{2}}(2 s) \sum_{n=1}^{\infty} \frac{a_{f}(n) \overline{a_{g}(n)} \chi_{1}(n) \overline{\chi_{2}(n)}}{n^{s}} \\
=\left(1-\psi_{1}(p) \overline{\psi_{2}(p)} \chi_{1}(p)^{2} \bar{\chi}_{2}(p) p^{2} p^{-2 s}\right)^{-1} \sum_{l=0}^{\infty} \frac{a_{f}\left(p^{l}\right) \overline{a_{g}\left(p^{l}\right)} \chi_{1}\left(p^{l}\right) \overline{\chi_{2}\left(p^{l}\right)}}{p^{l s}}=\left(L_{f, \chi_{1}} \otimes L_{g, \chi_{2}}\right)(s) .
\end{gathered}
$$

This completes the proof.

In our applications, we also need the following theorem of Rankin [11].

Theorem 1.5 (Rankin) Let $f \in S_{k}\left(N, \psi_{1}\right)$ and $g \in S_{k}\left(N, \psi_{2}\right)$. Let

$$
\Phi(s)=\left(\frac{2 \pi}{\sqrt{N}}\right)^{-2 s} \Gamma(s) \Gamma(s+k-1) L(f \otimes g, s) .
$$

Then both $L(f \otimes g, s)$ and $\Phi(s)$ are entire if $\psi_{1} \neq \psi_{2}$ or $\langle f, g\rangle=0$. Otherwise, for $N=1$ they are analytic everywhere except that $L(f \otimes g, s)$ has a simple pole at $s=1$ and $\Phi(s)$ has simple poles at points $s=0$ and 1 , and for $N>1$ both $L(f \otimes g, s)$ and $\Phi(s)$ are analytic except a simple pole at $s=1$.

\section{Mertens's Method}

In 1898 Mertens gave a proof for the non-vanishing of $\zeta(s)$ on the line $\operatorname{Re}(s)=1$. Mertens's proof depends upon the choice of a suitable trigonometric inequality. This line of proof is adaptable for establishing the non-vanishing of various $L$-functions. For example in [10], Rankin used this method to prove the non-vanishing of $L_{f}(s)$ on the line $\operatorname{Re}(s)=1, s \neq 1$, where $f$ is an eigenform for $\Gamma_{0}(N)$. Another example is the proof of the following lemma, due to K. Murty [7], which, similar to Mertens's proof, depends on a certain trigonometric inequality. 
Lemma 2.1 Let $f(s)$ be a complex function satisfying the following:

(i) $f(s)$ is analytic in $\operatorname{Re}(s)>1$ and non-zero there;

(ii) $\log f(s)$ can be written as a Dirichlet series

$$
\sum_{n=1}^{\infty} \frac{b_{n}}{n^{s}}
$$

with $b_{n} \geq 0$ for $\operatorname{Re}(s)>1$;

(iii) On the line $\operatorname{Re}(s)=1, f(s)$ is analytic except for a pole of order $e \geq 0$ at $s=1$.

Then, if $f(s)$ has a zero on the line $\operatorname{Re}(s)=1$, the order of that zero is bounded by $\frac{e}{2}$.

Proof See [7], Lemma 3.2.

Here by employing the above lemma we prove a conditional theorem regarding the nonvanishing of $(F \otimes G)(s)$ on the punctured line $\operatorname{Re}(s)=1(s \neq 1)$. The following definition describes one of the main conditions of our theorem.

Definition 2.2 For $F \in \mathcal{S}^{*}$ and $\sigma_{0} \leq 1$, we say $F$ is $\otimes$-simple in $\operatorname{Re}(s)>\sigma_{0}\left(\operatorname{resp} . \operatorname{Re}(s) \geq \sigma_{0}\right)$, if $F \otimes F$ has an analytic continuation to $\operatorname{Re}(s)>\sigma_{0}$ (resp. $\left.\operatorname{Re}(s) \geq \sigma_{0}\right)$, except for a possible simple pole at $s=1$.

The following theorem is the main result of this section.

Theorem 2.3 Let $F, G \in \mathcal{S}^{*}$ be $\otimes$-simple in $\operatorname{Re}(s) \geq 1$ and $t \neq 0$. Then

(i) $(F \otimes F)(1+i t) \neq 0$.

(ii) If $F \otimes G$ has an analytic continuation to the line $\operatorname{Re}(s)=1$ and $(F \otimes G)(s)=0$ if and only if $(F \otimes G)(\bar{s})=0$ for any $s$ on the line $\operatorname{Re}(s)=1$, then $(F \otimes G)(1+i t) \neq 0$.

Proof (i) Let $f(s)=(F \otimes F)(s)$. We have

$$
\log f(s)=\sum_{p} \sum_{k=1}^{\infty} \frac{k\left|b_{F}\left(p^{k}\right)\right|^{2}}{p^{k s}}=\sum_{n=1}^{\infty} \frac{c(n)}{n^{s}},
$$

with $c(n) \geq 0$. So, $f(s)$ satisfies the conditions of Lemma 2.1 with $e=1$. Therefore, the order of the vanishing of $f(s)$ at point $1+i t$ is $\leq \frac{1}{2}$. This means that $(F \otimes F)(1+i t) \neq 0$.

(ii) Let

$$
f(s)=(F \otimes F)(s)(F \otimes G)(s)(G \otimes F)(s)(G \otimes G)(s) .
$$

Since for $t \neq 0$, all the factors of $f(s)$ have finite values at point $1+i t$, in order to prove that $(F \otimes G)(1+i t) \neq 0$, it suffices to show that $f(1+i t) \neq 0$. Note that

$$
\log f(s)=\sum_{p} \sum_{k=1}^{\infty} \frac{k\left|b_{F}\left(p^{k}\right)+b_{G}\left(p^{k}\right)\right|^{2}}{p^{k s}}=\sum_{n=1}^{\infty} \frac{c(n)}{n^{s}}
$$


with $c(n) \geq 0$. So, $f(s)$ satisfies the conditions of Lemma 2.1 with $e \leq 2$, and therefore, the order of the vanishing of $f(s)$ at point $1+i t$ is $\leq 1$. Now suppose that $f(1+i t)=0$. Thus,

$$
(F \otimes F)(1+i t)(F \otimes G)(1+i t) \overline{(F \otimes G)(1-i t)}(G \otimes G)(1+i t)=0 .
$$

Since by part $(i),(F \otimes F)(1+i t) \neq 0$ and $(G \otimes G)(1+i t) \neq 0$, it follows that $(F \otimes G)(1+i t)=0$. This is a contradiction, otherwise, the order of the vanishing of $f(s)$ at point $1+i t$ should be 2 .

Note In Theorem 2.3 in fact we can have $(F \otimes G)(1)=0$. To see this, Let $F(s)=\sum_{n=1}^{\infty} \frac{(-1)^{\Omega(n)}}{n^{s}}$ and $G(s)=\zeta(s)$, where $\Omega(n)$ is the total number of prime factors of $n$. Then we have $(F \otimes$ $G)(s)=\frac{\zeta(2 s)}{\zeta(s)}$ and so $(F \otimes G)(1)=0$.

Corollary 2.4 Let $f \in S_{k}(N, \psi)$ be a normalized eigenform for $\Gamma_{0}(N)$ and let $t \neq 0$. Then

(i) $\zeta(1+i t) \neq 0$.

(ii) $L(f \otimes f, 1+i t) \neq 0$.

(iii) For trivial $\psi$ we have $L\left(\right.$ sym $\left.^{2} f, 1+i t\right) \neq 0$. Here $t$ is any real number including zero.

Proof (i) This is a consequence of part (i) of Theorem 2.3 with $F(s)=\zeta(s)$.

(ii) From part $(v)$ of Lemma 1.4 we have $\left(L_{f} \otimes L_{f}\right)(s)=L(f \otimes f, s)$. By Theorem 1.5 we know that $L(f \otimes f, s)$ is entire except a simple pole at $s=1$. Thus $L_{f}(s)$ is $\otimes$-simple in the whole plane. So $L_{f}(s)$ satisfies all the conditions of part (i) of Theorem 2.3 and we have

$$
L(f \otimes f, 1+i t)=\left(L_{f} \otimes L_{f}\right)(1+i t) \neq 0 .
$$

(iii) Note that $L\left(s^{2} m^{2} f, s\right)=L(f \otimes f, s) / \zeta_{N}(s)$. So the result follows from part (i) and (iii) for $t \neq 0$. For $t=0, L\left(\operatorname{sym}^{2} f, 1\right)$ in a non-zero multiple of $\langle f, f\rangle$ (see [11], Theorem 3 (iii)) and therefore it is non-vanishing.

Corollary 2.5 If $F=\bar{F} \in \mathcal{S}^{*}$ is analytic and $\otimes$-simple in $\operatorname{Re}(s) \geq 1$, then $F(1+i t) \neq 0$ for $t \neq 0$.

Proof This is a simple consequence of part (ii) of the previous theorem with $G(s)=\zeta(s)$.

Corollary 2.6 Let $f \in S_{k}(N)$ be an eigenform for $\Gamma_{0}(N)$, let $\chi$ be a real non-trivial Dirichlet character $(\bmod q)$, and let $t \neq 0$. Then

(i) $L_{\chi}(1+i t) \neq 0$.

(ii) $L_{f}(1+i t) \neq 0$.

(iii) $L_{f, \chi}(1+i t) \neq 0$.

(iv) Suppose $g \in S_{k}(N)$ is also an eigenform for $\Gamma_{0}(N)$. If $\langle f, g\rangle=0$, then $L(f \otimes g, 1+i t) \neq 0$. 
Proof Note that without loss of generality, we can assume that $f$ is normalized.

(i) By part (iii) of Lemma 1.4, $L_{\chi}(s)$ is $\otimes$-simple in the whole plane. Since $L_{\chi}(s)$ is analytic on the line $\operatorname{Re}(s)=1$, by Corollary 2.5 we have the desired results.

(ii) Part (v) of Lemma 1.4 and Theorem 1.5 imply that $L_{f}(s)$ is $\otimes$-simple. Since $L_{f}(s)$ is analytic on the line $\operatorname{Re}(s)=1$, by Corollary $2.5 L_{f}(1+i t) \neq 0$.

(iii) As we showed in part (i) and (ii) $L_{\chi}(s)$ and $L_{f}(s)$ are $\otimes$-simple. Now since $f$ is an eigenform and $\chi$ is real, the coefficients of $L_{f, \chi}(s)$ are real. Also $L_{f, \chi}(s)$ is the $L$-function associated to a cusp form of weight $k$ and level $q^{2} N$ (see [5], p. 127, Proposition 17 (b)). So, $L_{f, \chi}(s)$ is analytic on the line $\operatorname{Re}(s)=1$. Therefore, by part (iv) of Lemma 1.4 and part (ii) of Theorem 2.3,

$$
L_{f, \chi}(1+i t)=\left(L_{f} \otimes L_{\chi}\right)(1+i t) \neq 0 .
$$

(iv) Note that the coefficients of eigenforms are real and $L_{f}(s)$ and $L_{g}(s)$ are $\otimes$-simple in the whole plane. If $\langle f, g\rangle=0$, by Theorem $1.5 L(f \otimes g, s)$ is actually an entire function. Therefore, by part ( $v$ ) of Lemma 1.4 and part (ii) of Theorem 2.3, we have

$$
L(f \otimes g, 1+i t)=\left(L_{f} \otimes L_{g}\right)(1+i t) \neq 0 .
$$

This completes the proof.

\section{Ingham's Method}

One of the main facts regarding Dirichlet series with positive coefficients is the following result of Landau.

Lemma 3.1 (Landau) A Dirichlet series with non-negative coefficients has a singularity at its abscissa of convergence.

Proof See [6], Exercise 2.5.14.

In this section, we will show that for two Dirichlet series in $\mathcal{S}^{*}$ with completely multiplicative coefficients $^{2}$, one can apply this lemma of Landau to prove a non-vanishing result, valid on the line $\operatorname{Re}(s)=1$, for the convolution series. Our result is a generalization of Ingham's proof of the non-vanishing of the Riemann zeta-function on the line $\operatorname{Re}(s)=1$ [3]. To do this, we start with recalling some results regarding Dirichlet series with completely multiplicative coefficients and completely multiplicative arithmetic functions.

\footnotetext{
${ }^{2}$ This means $a_{F}(m n)=a_{F}(m) a_{F}(n)$ for every $m$ and $n$.
} 
Lemma 3.2 For $F, G \in \mathcal{S}^{*}$ with completely multiplicative coefficients,

$$
(F \otimes G)(s)=\sum_{n=1}^{\infty} \frac{a_{F}(n) \overline{a_{G}(n)}}{n^{s}} .
$$

Proof We have

$$
(F \otimes G)(s)=\prod_{p} \exp \left(\sum_{k=1}^{\infty} \frac{a_{F}(p)^{k}\left(\overline{a_{G}(p)}\right)^{k} / k}{p^{k s}}\right)=\prod_{p}\left(1-a_{F}(p) \overline{a_{G}(p)} p^{-s}\right)^{-1}=\sum_{n=1}^{\infty} \frac{a_{F}(n) \overline{a_{G}(n)}}{n^{s}} .
$$

Definition 3.3 If $f(n)$ is an arithmetic function, the formal L-series attached to $f(n)$ is defined by

$$
L(f, s)=\sum_{n=1}^{\infty} \frac{f(n)}{n^{s}} .
$$

If $g(n)$ is also an arithmetic function, the Dirichlet convolution of $f(n)$ and $g(n)$ is defined by

$$
(f * g)(n)=\sum_{d \mid n} f(d) g\left(\frac{n}{d}\right)
$$

The following identity of formal $L$-series, due to J. Borwein and Choi [1], will be fundamental in the proof of the main result of this section.

Lemma 3.4 Let $f_{1}, f_{2}, g_{1}, g_{2}$ be completely multiplicative arithmetic functions. Then we have

$$
\sum_{n=1}^{\infty} \frac{\left(f_{1} * g_{1}\right)(n)\left(f_{2} * g_{2}\right)(n)}{n^{s}}=\frac{L\left(f_{1} f_{2}, s\right) L\left(g_{1} g_{2}, s\right) L\left(f_{1} g_{2}, s\right) L\left(f_{2} g_{1}, s\right)}{L\left(f_{1} f_{2} g_{1} g_{2}, 2 s\right)}
$$

Proof See [1], Theorem 2.1.

We are ready to state and prove the main result of this section.

Theorem 3.5 Let $F, G \in \mathcal{S}^{*}$ be two Dirichlet series with completely multiplicative coefficients.

Also assume the following:

(i) $F$ and $G$ are $\otimes$-simple in $\operatorname{Re}(s)>\frac{1}{2}$;

(ii) $F \otimes G$ has an analytic continuation to $\operatorname{Re}(s)>\frac{1}{2}$;

(iii) $(F \otimes G) \otimes(F \otimes G)$ is analytic for $\operatorname{Re}(s)>1$ and has a pole at $s=1$.

(iv) $(F \otimes F)(s),(G \otimes G)(s)$ and $(F \otimes G)(s)$ have finite limits as $s \rightarrow \frac{1}{2}^{+} 3$. Then, $(F \otimes G)(1+i t) \neq 0$ for all $t$.

\footnotetext{
${ }^{3}$ This means $s=\sigma+i t \rightarrow \frac{1}{2}+i t$ for any $t$ as $\sigma \rightarrow \frac{1}{2}^{+}$.
} 
Proof Let

$$
F(s)=\sum_{n=1}^{\infty} \frac{a_{F}(n)}{n^{s}}, G(s)=\sum_{n=1}^{\infty} \frac{a_{G}(n)}{n^{s}}
$$

and suppose that $(F \otimes G)\left(1+i t_{0}\right)=0$ for a real $t_{0}$. Let

$$
f_{1}(n)=a_{F}(n) n^{-i t_{0}}, f_{2}(n)=\overline{a_{F}(n)} n^{i t_{0}}, g_{1}(n)=a_{G}(n), g_{2}(n)=\overline{a_{G}(n)},
$$

and for $\operatorname{Re}(s)>1$, consider the following Dirichlet series

$$
f(s)=\sum_{n=1}^{\infty} \frac{\left|\left(f_{1} * g_{1}\right)(n)\right|^{2}}{n^{s}}=\sum_{n=1}^{\infty} \frac{\left(f_{1} * g_{1}\right)(n)\left(f_{2} * g_{2}\right)(n)}{n^{s}} .
$$

Since $f_{1}$ and $f_{2}$ are completely multiplicative, by Lemma 3.2 we have

$$
L\left(f_{1} f_{2}, s\right)=\sum_{n=1}^{\infty} \frac{\left|a_{F}(n)\right|^{2}}{n^{s}}=(F \otimes F)(s) .
$$

Similarly, we can derive the following

$$
L\left(g_{1} g_{2}, s\right)=(G \otimes G)(s), \quad L\left(f_{1} g_{2}, s\right)=(F \otimes G)\left(s+i t_{0}\right), \quad L\left(f_{2} g_{1}, s\right)=(G \otimes F)\left(s-i t_{0}\right),
$$

and

$$
L\left(f_{1} f_{2} g_{1} g_{2}, 2 s\right)=[(F \otimes G) \otimes(F \otimes G)](2 s) .
$$

So, by Lemma 3.4 and for $\operatorname{Re}(s)>1$, we have

$$
f(s)=\frac{(F \otimes F)(s)(F \otimes G)\left(s+i t_{0}\right)(G \otimes F)\left(s-i t_{0}\right)(G \otimes G)(s)}{[(F \otimes G) \otimes(F \otimes G)](2 s)} .
$$

Now by assumption of $(F \otimes G)\left(1+i t_{0}\right)=0$ we have in fact the analyticity of $f(s)$ for $\operatorname{Re}(s)>\frac{1}{2}$, and since the coefficients in the series are non-negative, by Lemma 3.1 the Dirichlet series representing $f(s)$ is convergent for $\operatorname{Re}(s)>\frac{1}{2}$. So, for $\eta>0$, we have

$$
f\left(\frac{1}{2}+\eta\right)=\sum_{n=1}^{\infty} \frac{\left|\left(f_{1} * g_{1}\right)(n)\right|^{2}}{n^{\frac{1}{2}+\eta}} \geq 1 .
$$

However, since $(F \otimes G) \otimes(F \otimes G)$ has a pole at $s=1$,

$$
[(F \otimes G) \otimes(F \otimes G)]\left(2\left(\frac{1}{2}+\eta\right)\right)=[(F \otimes G) \otimes(F \otimes G)](1+2 \eta) \rightarrow \infty
$$

as $\eta \rightarrow 0^{+}$. This shows that

$$
\lim _{\eta \rightarrow 0^{+}} f\left(\frac{1}{2}+\eta\right)=0
$$

which is a contradiction. 
By choosing $G(s)=\zeta(s)$ in the previous theorem, we have

Corollary 3.6 Let $F \in \mathcal{S}^{*}$ be analytic and $\otimes$-simple in $\operatorname{Re}(s)>\frac{1}{2}$. If the coefficients of $F$ are completely multiplicative and $F(s)$ together with $(F \otimes F)(s)$ have finite limits as $s \rightarrow \frac{1}{2}^{+}$, then $F(1+i t) \neq 0$, for all $t \in \mathbb{R}$.

The following non-vanishing results are simple consequences of the previous corollary.

Corollary 3.7 Let $\chi$ be a non-trivial Dirichlet character and let $f(n)$ be a completely additive ${ }^{4}$ arithmetic function and let $t \in \mathbb{R}$. Then

(i) $L_{\chi}(1+i t) \neq 0$.

(ii) If $\sum_{n \leq x}(-1)^{f(n)} \chi(n)=O\left(x^{\delta}\right)$ for $\delta<\frac{1}{2}$, then $L(s)=\sum_{n=1}^{\infty} \frac{(-1)^{f(n)} \chi(n)}{n^{s}}$ is analytic in $\operatorname{Re}(s)>\delta$ and $L(1+i t) \neq 0$.

\section{Ogg's Method}

In section 2, we proved a general non-vanishing result on the line $\operatorname{Re}(s)=1$, however our result were applicable mostly for Dirichlet series with real coefficients and also it did not cover the point $s=1$. In the previous section we overcome these difficulties for the case of Dirichlet series with completely multiplicative coefficients. In this section, we consider the extension of the results of Section 3 to Dirichlet series with general coefficients. Our approach in this section is motivated by a paper of Ogg [9]. The following lemma describes the basic ingredient of this approach.

Lemma 4.1 Let $f(s)$ be a complex function that satisfies the following:

(i) $f(s)$ is analytic on the half-plane $\operatorname{Re}(s)>\sigma_{0}$;

(ii) $\log f(s)$ has a representation in terms of a Dirichlet series with non-negative coefficients on the half-plane $\operatorname{Re}(s)>\sigma_{1}\left(\sigma_{1}>\sigma_{0}\right)$.

Then $f(s) \neq 0$ for $\operatorname{Re}(s)>\sigma_{0}$.

Proof Let $\sigma_{2}$ be the largest real zero of $f\left(\sigma_{0}<\sigma_{2} \leq \sigma_{1}\right)$. Since $\log f(s)=\sum_{n=1}^{\infty} \frac{c(n)}{n^{s}}$ for $\operatorname{Re}(s)>\sigma_{1}(c(n) \geq 0)$, and since $\log f(s)$ is analytic in a neighborhood of the segment $\sigma_{2}<\sigma \leq \sigma_{1}$, then by Lemma 3.1, we have $\log f(s)=\sum_{n=1}^{\infty} \frac{c(n)}{n^{s}}$ for $\operatorname{Re}(s)>\sigma_{2}$. Thus,

$$
\log |f(\sigma)|=\operatorname{Re}(\log f(\sigma))=\log f(\sigma)=\sum_{n=1}^{\infty} \frac{c(n)}{n^{\sigma}} \geq 0
$$

\footnotetext{
${ }^{4}$ This means $f(m n)=f(m) f(n)$ holds for all $m$ and $n$.
} 
for $\sigma>\sigma_{2}$. Therefore, $|f(\sigma)| \geq 1$ for $\sigma>\sigma_{2}$. This contradicts the assumption $f\left(\sigma_{2}\right)=0$, and therefore $f$ has no real zero $\sigma>\sigma_{0}$. So $\log f(s)$ is analytic on the interval $\left(\sigma_{0}, \sigma_{1}\right]$, and Lemma 3.1 in fact shows that $\log f(s)$ exists and is analytic for $\operatorname{Re}(s)>\sigma_{0}$. This means that $f(s)$ is non-zero for $\operatorname{Re}(s)>\sigma_{0}$.

Here, we prove the main result of this section.

Theorem 4.2 Let $\sigma_{0}<1$, and assume the following:

(i) $F$ and $G$ (as elements of $\mathcal{S}^{*}$ ) are $\otimes$-simple in $\operatorname{Re}(s)>\sigma_{0}$;

(ii) $F \otimes G$ has an analytic continuation to the half-plane $\operatorname{Re}(s)>\sigma_{0}$;

(iii) At least one of $F \otimes F, G \otimes G$, or $F \otimes G$ has zeros in the strip $\sigma_{0}<\operatorname{Re}(s)<1$. Then $(F \otimes G)(1+i t) \neq 0$ for all real $t$.

Proof Suppose that $(F \otimes G)\left(1+i t_{0}\right)=0$, and let

$$
f(s)=(F \otimes F)(s)(F \otimes G)\left(s+i t_{0}\right)(G \otimes F)\left(s-i t_{0}\right)(G \otimes G)(s) .
$$

First of all note that $G \otimes F$ is analytic for $\operatorname{Re}(s)>\sigma_{0}$. Since $(F \otimes G)\left(1+i t_{0}\right)=0$, then $(G \otimes F)\left(1-i t_{0}\right)=0$, and since $s=1$ is a pole of order $\leq 1$ for both $F \otimes F$ and $G \otimes G$, we conclude that $f(s)$ is analytic at point $s=1$, and therefore, analytic for $\operatorname{Re}(s)>\sigma_{0}$. Now note that for $\operatorname{Re}(s)>1$,

$$
\log f(s)=\sum_{p} \sum_{k=1}^{\infty} \frac{k\left|b_{F}\left(p^{k}\right)+b_{G}\left(p^{k}\right) p^{i k t_{0}}\right|^{2}}{p^{k s}}=\sum_{n=1}^{\infty} \frac{c(n)}{n^{s}}
$$

where $c(n) \geq 0$. So, $f(s)$ satisfies the conditions of the Corollary 4.1 with $\sigma_{1}=1$, and therefore, $f(s) \neq 0$ for $\operatorname{Re}(s)>\sigma_{0}$. This contradicts our assumption in (iii).

The following corollary gives an extension of Corollary 3.6 to the Dirichlet series with general coefficients.

Corollary 4.3 Let $F \in \mathcal{S}^{*}$ be analytic and $\otimes$-simple in $\operatorname{Re}(s) \geq \frac{1}{2}$, then $F(1+i t) \neq 0$.

Proof Let $G(s)=\zeta(s)$. Note that $F \otimes G=F$ and note that $\zeta(s)$ has zeros in the half-plane $\operatorname{Re}(s) \geq 1 / 2$ (see [2], p. 97). Thus, $F(1+i t)=(F \otimes G)(1+i t) \neq 0$.

Corollary 4.4 Let $f \in S_{k}\left(N, \psi_{1}\right)$ and $g \in S_{k}\left(N, \psi_{2}\right)$ be eigenforms for $\Gamma_{0}(N)$, let $\chi$ be a nontrivial Dirichlet character $(\bmod q)$ and let $t$ be any real number. Then

(i) $L_{\chi}(1+i t) \neq 0$.

(ii) $L_{f}(1+i t) \neq 0$. 
(iii) $L_{f, \chi}(1+i t) \neq 0$.

(iv) If $\psi_{1} \neq \psi_{2}$ or $\langle f, g\rangle=0$, then $L(f \otimes g, 1+i t) \neq 0$.

(v) Let $\bar{f}_{\bar{\chi}}(z)=\sum_{n=1}^{\infty} \overline{a_{f}(n) \chi(n)} e^{2 \pi i n z}$. Then if $\psi \chi$ is not a real character of order 2 or $\int_{D_{0}\left(N q^{2}\right)} f(z) \bar{f}_{\bar{\chi}}(z) y^{k-2} d x d y=0$, we have $L_{\chi}(f \otimes \bar{f}, 1+i t) \neq 0$ and $L_{\chi}\left(\operatorname{sym}^{2} f, 1+i t\right) \neq 0$. Here $D_{0}\left(N q^{2}\right)$ is a fundamental domain for $\Gamma_{0}\left(N q^{2}\right)$.

Proof (i), (ii) Both are simple consequences of Corollary 4.3. Note that $L_{\chi}(s)$ and $L_{f}(s)$ are entire and $\otimes$-simple in the whole plane.

(iii) Note that $L_{\bar{\chi}}(s)$ and $L_{f}(s)$ are $\otimes$-simple. Also by part (iv) of Lemma 1.4, we have $L_{f, \chi}(s)=\left(L_{f} \otimes L_{\bar{\chi}}\right)(s)$. We know that $L_{f, \chi}(s)$ is a cusp form of weight $k$, level $N q^{2}$ and character $\psi_{1} \chi^{2}$ (see [5], p. 127), so $\left(L_{f} \otimes L_{\chi}\right)(s)$ is entire. Also note that $\left(L_{\bar{\chi}} \otimes L_{\bar{\chi}}\right)(s)=\zeta_{q}(s)$ has in fact infinitely many zeros (see [2], p. 97). So, all the conditions of Theorem 4.2 are met and therefore, $L_{f, \chi}(1+i t)=\left(L_{f} \otimes L_{\bar{\chi}}\right)(1+i t) \neq 0$.

(iv) By Theorem 1.5 we can show that conditions (i) and (ii) of Theorem 4.2 are satisfied. The result will be obtained if we only show that $L(f \otimes g, s)$ has a zero in the half-plane $\operatorname{Re}(s)<1$. Again by Theorem 1.5, if $\psi_{1} \neq \psi_{2}$ or $\langle f, g\rangle=0$, then

$$
\Phi(s)=\left(\frac{2 \pi}{\sqrt{N}}\right)^{-2 s} \Gamma(s) \Gamma(s+k-1) L(f \otimes g, s)
$$

is analytic at $s=0$. Since $\Gamma(s)$ has a pole at $s=0$, then $L(f \otimes g, 0)=0$.

(v) First of all note that $\bar{f}_{\bar{\chi}} \in S_{k}\left(N q^{2}, \psi \chi^{2}\right)$ (see [5], p. 127) and $L_{\chi}(f \otimes \bar{f}, s)=L\left(f \otimes \bar{f}_{\bar{\chi}}, s\right)$. So under the given conditions, by part (iv) we have $L_{\chi}(f \otimes \bar{f}, 1+i t) \neq 0$. This together with part (i) imply that $L_{\chi}\left(s y m^{2} f, 1+i t\right)=L_{\chi}(f \otimes \bar{f}, 1+i t) / L_{\psi \chi}(1+i t) \neq 0$.

\section{References}

[1] J. M. Borwein and K. S. Choi, On Dirichlet series for sums of squares, The Ramanujan Journal, special issue for Robert Rankin 7 (2003), 29 pages.

[2] H. Davenport, Multiplicative number theory, Third ed., Springer-Verlag, 2000.

[3] A. E. Ingham, Note on Riemann's $\zeta$-function and Dirichlet's L-functions, JLMS 5 (1930), $107-112$.

[4] H. Jacquet and J. A. Shalika, A non-vanishing theorem for zeta functions of $G L_{n}$, Inventiones Math. 38 (1976), 1-16.

[5] N. Koblitz, Introduction to elliptic curves and modular forms, Second ed., Springer-Verlag, 1993. 
[6] M. R. Murty, Problems in analytic number theory, Springer-Verlag, 2001.

[7] V. K. Murty, On the Sato-Tate conjecture, Progress in Mathematics 26 (1982), 195-205.

[8] S. Narayanan, On the non-vanishing of a certain class of Dirichlet series, Canad. Math. Bull. 40 (1997), 364-369.

[9] A. P. Ogg, On a convolution of L-series, Inventiones Math. 7 (1969), 297-312.

[10] R. A. Rankin, Contributions to the theory of Ramanujan's function $\tau(n)$ and similar arithmetical functions, Proc. Camb. Phil. Soc. 35 (1939), 351-356.

[11] Contributions to the theory of Ramanujan's function $\tau(n)$ and similar arithmetical functions II, Proc. Camb. Phil. Soc. 35 (1939), 357-372.

[12] F. Shahidi, On non-vanishing of L-functions, Bull. of AMS 2 (1980), 462-464.

[13] _ On non-vanishing of twisted symmetric and exterior square L-functions for $G L(n)$, Pacific J. of Math. 181 (1997), 311-322.

Department of Mathematics and Computer Science, University of Lethbridge, 4401 University Drive West, Lethbridge, AB, T1K 3M4, CANADA

E-mail: akbary@cs.uleth.ca

E-mail: shahabi@cs.uleth.ca 